\title{
CXXC5 is a negative-feedback regulator of the Wnt/ $\beta$-catenin pathway involved in osteoblast differentiation
}

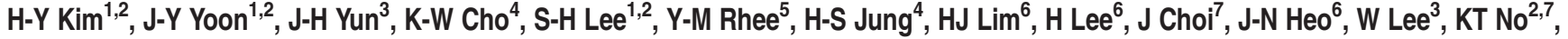 \\ D Min ${ }^{1,8}$ and K-Y Choi, ${ }^{*, 1,2}$
}

The positive roles of the Wnt/ $\beta$-catenin pathway in osteoblast differentiation and bone mineral density (BMD) maintenance have been clearly demonstrated in both animal experiments and clinical investigations. CXXC finger protein 5 (CXXC5), a recently identified negative regulator of the Wnt/ $\beta$-catenin pathway, showed altered cellular localization and function, which were dependent on the cell type in previous studies. However, the in vivo function of CXXC5 has not been clearly investigated yet. Here, we characterized CXXC5 as a negative regulator of osteoblast differentiation and bone formation. Deficiency of CXXC5 resulted in elevated BMD in mice without any severe gross developmental abnormalities. CXXC5 exerted a negative-feedback effect on the Wnt $\beta$-catenin pathway via Wnt-dependent binding to Dishevelled (Dvl) during osteoblast differentiation. Suppression of the Dvl-CXXC5 interaction using a competitor peptide resulted in the activation of the Wnt/ $\beta$-catenin pathway and osteoblast differentiation, and accelerated thickness growth of ex vivo-cultured calvariae. Overall, CXXC5 is a negative-feedback regulator induced by Wnt/ $\beta$-catenin signaling that inhibits osteoblast differentiation and bone formation via interaction with Dvl.

Cell Death and Differentiation (2015) 22, 912-920; doi:10.1038/cdd.2014.238; published online 30 January 2015

Bone is an extremely dynamic tissue at the microscopic level. A dynamic process, called bone remodeling, takes place seamlessly in the bone to repair microdamage and to replace old bone with new bone. ${ }^{1}$ The resorption of old bone and the formation of new bone must be in balance to maintain homeostasis and a constant mass of bone. Osteoblasts have been identified as an essential factor in regulation of the bone remodeling process, which produce bone matrix and differentiate into osteocytes for bone formation, as well as regulate differentiation and activation of osteoclasts for bone resorption. ${ }^{2}$

The Wnt/ $\beta$-catenin pathway is receiving increased attention as a main regulatory pathway for osteoblast differentiation. ${ }^{2-4}$ Wnt-dependent nuclear accumulation of an effector protein of the pathway, $\beta$-catenin, is a major trigger of osteoblast differentiation and bone formation. ${ }^{2}$ Many other intracellular and extracellular components of the $\mathrm{Wnt} / \beta$-catenin pathway are known to regulate osteoblast differentiation. ${ }^{4}$ Especially two negative regulators of this pathway, Dickkopf 1 and sclerostin, have been highlighted as osteoblast and osteocytespecific negative regulators of bone formation. ${ }^{5}$

CXXC finger protein 5 (CXXC5) is a member of a small protein family in which the members contain CXXC-type zincfinger domain. ${ }^{6}$ However, unlike other members of this family, CXXC5 lacks a KFGG motif, which is essential for non-methylated $\mathrm{CpG}$ recognition that regulates chromatin remodeling. ${ }^{7}$ CXXC5 localizes to the cytosol or nucleus depending on particular cell type in different tissues. Localization of CXXC5 in the nucleus was observed in promyelocytic leukemia cells. ${ }^{7} \mathrm{CXXC5}$ has a role as a nuclear transcription activator for Flk-1 and stimulates the embryonic endothelial differentiation in murine embryonic stem cells (mESCs). ${ }^{8}$ Meanwhile, CXXC5 localized at the cytosol of neural stem cells and interacts with Dishevelled (Dvl) to inhibit the Wnt/ $\beta$-catenin pathway ${ }^{9}$ similar to another CXXC-type zinc-finger protein lacking the KFGG motif, Idax (CXXC4). ${ }^{10}$ However, most experiments were performed using in vitro cell culture systems, and no clear evidence for the in vivo function of CXXC5 has been suggested yet. In this study, we found that CXXC5 localizes to the cytosol of pre-osteoblasts. CXXC5 functions as a negative-feedback regulator of the Wnt/ $\beta$-catenin pathway inhibiting osteoblast differentiation via Wnt3a-dependent binding to Dvl. CXXC5 knockout resulted in increased bone mineral density (BMD) and trabecular number in mice, which reveals a possible role for the DvlCXXC5 interaction in the regulation of bone formation. The role of this interaction was confirmed by experiments using a competitor peptide of the Dvl-CXXC5 interaction, which induced osteoblast differentiation and enhanced bone formation in ex vivo-cultured calvariae. Overall, we suggest that CXXC5 is a negative-feedback regulator of bone formation,

\footnotetext{
${ }^{1}$ Translational Research Center for Protein Function Control, Yonsei University, Seoul, Korea; ${ }^{2}$ Department of Biotechnology, College of Life Science and Biotechnology, Yonsei University, Seoul, Korea; ${ }^{3}$ Department of Biochemistry, College of Life Science and Biotechnology, Yonsei University, Seoul, Korea; ${ }^{4}$ Department of Oral Biology, Research Center for Orofacial Hard Tissue Regeneration, College of Dentistry, Yonsei University, Seoul, Korea; ${ }^{5}$ Department of Internal Medicine, Yonsei University College of Medicine, Seoul, Korea; ${ }^{6}$ Korea Research Institute of Chemical Technology, Daejeon, Korea; ${ }^{7}$ Bioinformatics and Molecular Design Research Center, Yonsei University, Seoul, Korea and ${ }^{8}$ Department of Molecular Biology, College of Natural Science, Pusan National University, Busan, Korea

${ }^{*}$ Corresponding author: K-Y Choi, Department of Biotechnology, College of Life Science and Biotechnology, Yonsei University, Seoul 120-749, Korea. Tel: +82 22123 2887; Fax: +82 22123 8284; E-mail: kychoi@yonsei.ac.kr

Abbreviations: $\alpha$-MEM, $\alpha$-minimal essential medium; ALP, alkaline phosphatase; BMD, bone mineral density; BSP, bone sialoprotein; CT, computed tomography; DAB, 3,3'-diaminobenzidine; DBM, Dvl-binding motif; DMP-1, dentin matrix acidic phosphoprotein-1; Dvl, Dishevelled; Fgf18, fibroblast growth factor 18; H\&E, hematoxylin and eosin; PTD, protein transduction domain

Received 03.7.14; revised 12.12.14; accepted 17.12.14; Edited by H-U Simon; published online 30.1.15
} 
and that the Dvl-CXXC5 interaction is an essential regulatory mechanism of bone formation via the $\mathrm{Wnt} / \beta$-catenin pathway.

\section{Results}

$\mathrm{CXXC5}^{-1}$ mice show high bone mass phenotypes. CXXC5 is evolutionary conserved among vertebrates from zebrafish to humans (Supplementary Figure S1A). In adult mice, CXXC5 mRNA and protein were expressed in several tissues, including muscle, pancreas, colon, skin and bone (Supplementary Figures S1B and C). To identify the in vivo role of $\mathrm{CXXC5}$, we generated $\mathrm{CXXC5} 5^{1-}$ mice by homologous recombination, replacing exon 2 (which contains the ATG start codon) with the phosphoglycerate kinase gene promoter and the neomycin resistance gene (PGK-Neo; Figure 1a). Disruption of the CXXC5 gene in mESCs was confirmed by Southern blot analysis (Supplementary Figure S2A), and the $C X X C 5$ deletion was confirmed in mice by PCR analysis of tail genomic DNA (Supplementary Figure S2B). CXXC5 knockout was also confirmed by immunohistochemical analysis of adult mouse tissues including kidney tissue; the level of CXXC4, the CXXC5 analog known as Idax, ${ }^{10}$ was not altered in $C X X C 5^{-1-}$ mice (Supplementary Figure $\mathrm{S} 2 \mathrm{C}$ ).

$\mathrm{CXXC5}{ }^{-1-}$ mice were born at the expected Mendelian ratios and no severe gross developmental abnormalities were observed in these mice. Interestingly, the skeleton of 11-week-

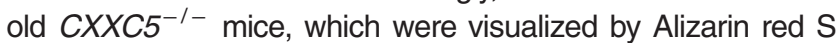
staining, showed enlargements of the skull, scapula, spine, ribs and limb bones compared with wild-type mice (Figure 1b). For quantitative analysis, BMDs of the 11-week-old mice were measured using dual-energy X-ray absorptiometry (Figures 1c-e). Average whole-body BMDs increased by $8.9 \%$ in $C X X C 5^{-1-}$ mice compared with wild-type and $C X X C 5^{+/-}$mice (Figure 1d). Average femoral BMDs increased by $20.0 \%$ in $C X X C 5^{-1-}$ mice compared with wild-type mice (Figure 1e).

Three-dimensional (3D) micro-computed tomography (CT) demonstrated that tibiae, fibulae, metatarsals and digits of 11week-old $C X X C 5^{-1-}$ mice were larger than those of wild-type mice (Figure 2a). Horizontal sections of the midpoints of the

a

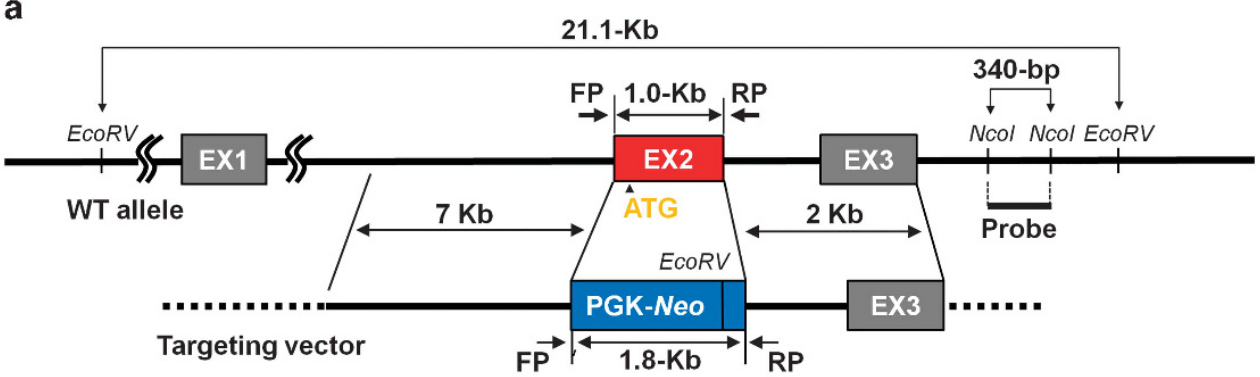

b

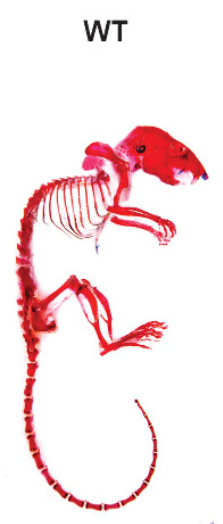

C

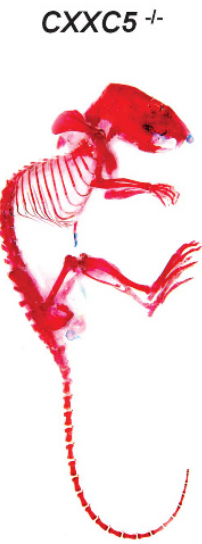

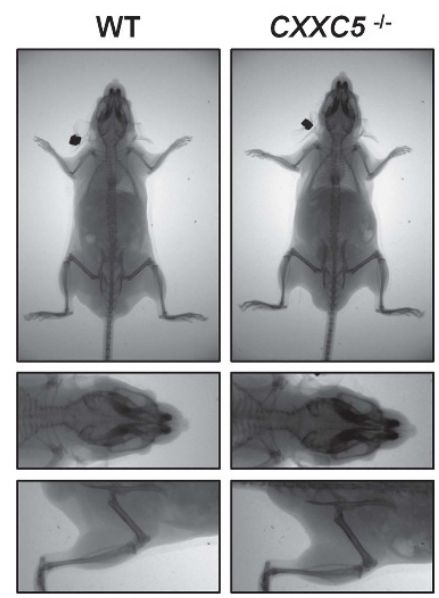

d

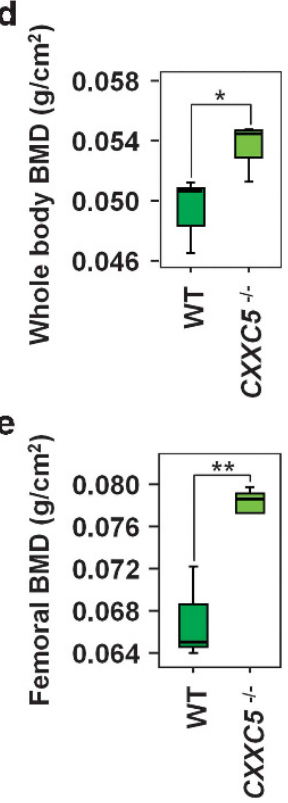

Figure 1 High bone mass phenotype of $C X X C 5^{-1-}$ mice. (a) Scheme of $C X X C 5$ knockout by homologous recombination. The upper line represents the structure of $C X X C 5$ in the wild-type mouse chromosome. The lower line represents a gene map of the targeting vector (pPNT-CXXC5) that was used in the generation of the CXXC5 knockout mice. Oblique lines between the two indicate the recombination scheme. After recombination, PGK-Neo was substituted for exon 2, resulting in CXXC5 knockout. The positions of the forward and reverse primers (FP and RP) for mouse genotyping are indicated. (b) Alizarin red-stained skeletons of 11-week-old WTand CXXC5 ${ }^{-/-}$mice. (c-e) BMDs and body weights of ten 11-week-old mice (four and six mice for WTand $C X X C 5^{-1-}$, respectively) were measured as describe in Materials and Methods section. The representative X-ray images of each genotype are shown in c. Graphs showing whole-body BMDs (d) and femoral BMDs (e). The box plots show the 75th and 25th percentiles, and the whiskers show the 10th and 90th percentiles. Significance was assessed using Kruskal-Wallis test; ${ }^{*} P<0.05$ and ${ }^{* *} P<0.01$ 
a

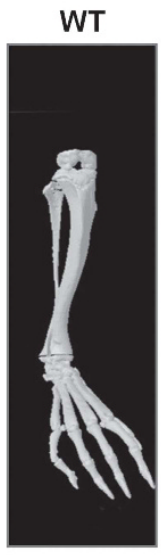

b

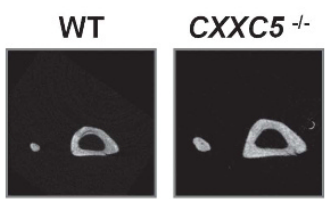

C

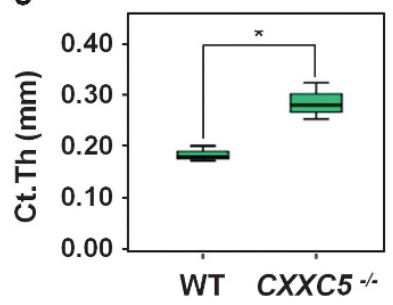

d
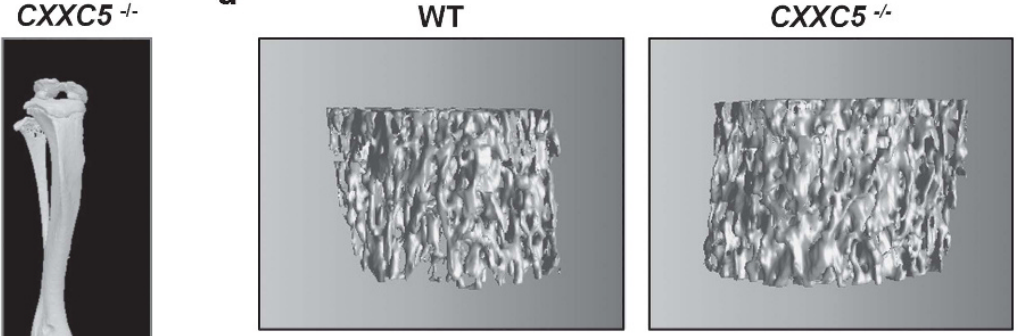

e

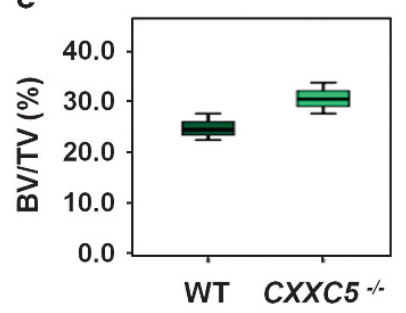

$\mathbf{f}$

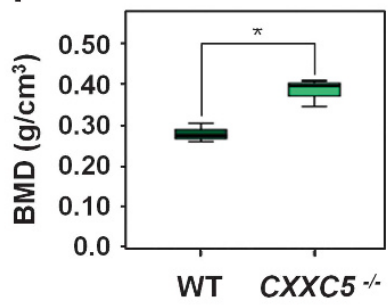

h

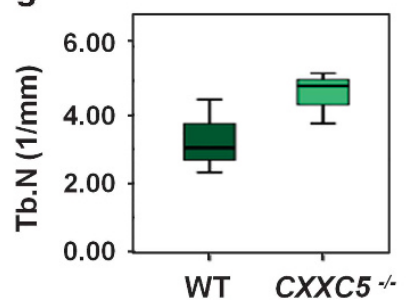

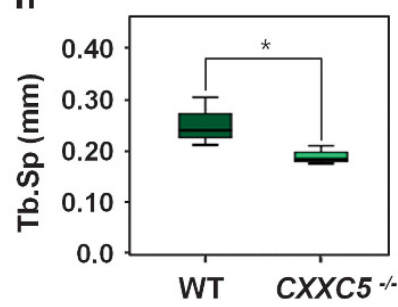

Figure 2 Increase of lower limb bones in $C X X C 5^{-1-}$ mice. $(\mathbf{a}-\mathbf{h})$ The lower limb bones of mice $(n=4)$ were scanned using micro-CT. Three-dimensional images of tibia, fibula, metatarsals and digits (a), and horizontal sections of midpoint of the tibia and fibula (b) of the WTand $C X X C 5^{-1-}$ mice were visualized. The cortical bone thicknesses of tibia (Ct.Th; c) were calculated by micro-CT scanning. Femoral trabecular bones were reconstructed (d) and the bone volume density (BV/TV; e), BMD (f), trabecular number (Tb.N; g) and trabecular separations (Tb.Sp; $\mathbf{h}$ ) were calculated from the micro-CT data. The box plots show the 75th and 25th percentiles, and the whiskers show the 10th and 90th percentiles. Significance was assessed using Kruskal-Wallis test; ${ }^{*} P<0.05$

tibia and fibula, visualized by micro-CT scanning, revealed significantly increased cortical bone thickness of tibiae and fibulae in the $C X X C 5^{-1-}$ mice (Figures $2 \mathrm{~b}$ and $\mathrm{c}$ ). These analyses also revealed the tendency of the femoral bone volume density to increase and a significant increase in the BMD of $C X X C 5^{-1-}$ mice compared with wild-type littermates (Figures 2d-f). The number of femoral trabecular bone also showed a tendency to increase, whereas separations between trabecular bones decreased significantly in $\mathrm{CXXC5}^{-/-}$mice (Figures 2d, g and h).

$\mathrm{CXXC5}^{-1}$ mice show increased osteocyte dendrite formation and bone formation rate. To further investigate the bone phenotype of $C X X C 5^{\prime-}$ mice, the tibiae of wildtype and $C X X C 5^{-1-}$ mice were subjected to histological analyses. The increments of length and thickness of tibiae in $C X X C 5^{-1-}$ mice were revealed by the hematoxylin and eosin (H\&E) staining of longitudinal sections of the tibiae (Figures $3 a$ and $b$ ). Dentin matrix acidic phosphoprotein-1 (DMP-1) is an important osteocyte marker that regulates the biomineralization activity and dendritic outgrowth of osteocytes. ${ }^{11}$ The numbers of $\beta$-catenin- and DMP-1-positive cells were higher in femoral sections from $C X X C 5^{-1-}$ mice than those from wild-type mice (Figure 3c). Furthermore, DMP-1 expression levels were higher in the osteocytes of $C X X C 5^{-1-}$ mice (Supplementary Figure S3A, and Figures $3 c$ and $d$ ). The number and length of osteocyte dendrites, which are essential for osteocytes to function as regulators of bone turnover and mechanosensors, ${ }^{12}$ were also higher in $C X X C 5^{-1-}$ mice than in wild-type mice (Figures $3 \mathrm{~d}-\mathrm{f}$, and Supplementary Figure S3B). Calcein double-labeling analyses ${ }^{13}$ showed that bone formation by osteoblasts was accelerated in the femur of $C X X C 5^{-1-}$ mice (Figures $3 \mathrm{~g}$ and $\mathrm{h}$, and Supplementary Figures S3A and B). These results show that $C X X C 5$ deficiency resulted in the enhancement of osteocyte functions, which could be the consequential results of the activation of osteoblasts differentiation.

CXXC5 acts as a negative-feedback regulator of the Wnt/ $\beta$-catenin pathway and osteoblast differentiation via its transcriptional induction and subsequent enhancement of Dvl interactions in a Wnt3a-dependent manner. The function of CXXC5 in osteoblast differentiation was investigated using MC3T3E1 cells (a murine pre-osteoblast cell line).$^{14}$ Interestingly, during cellular differentiation, the expression kinetics of both the CXXC5 mRNA and protein levels 
a

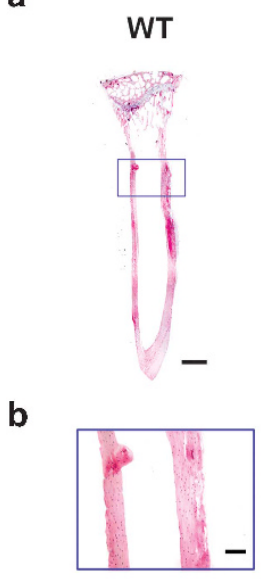

e

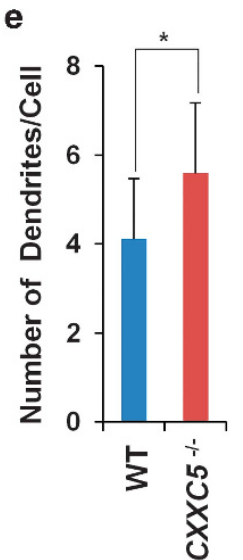

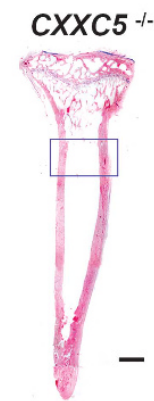

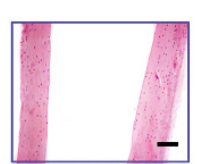

f

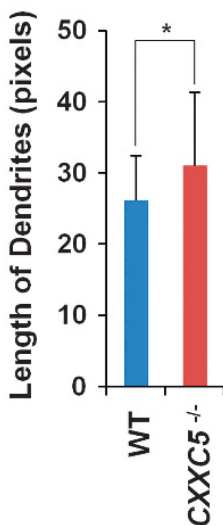

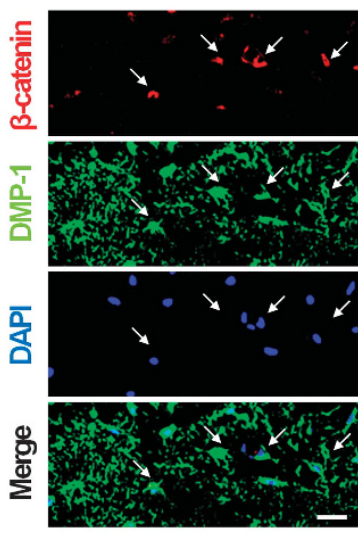

g

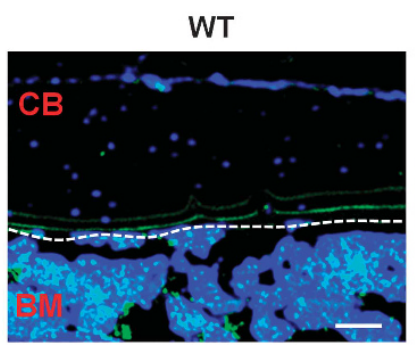

CXXC5\%

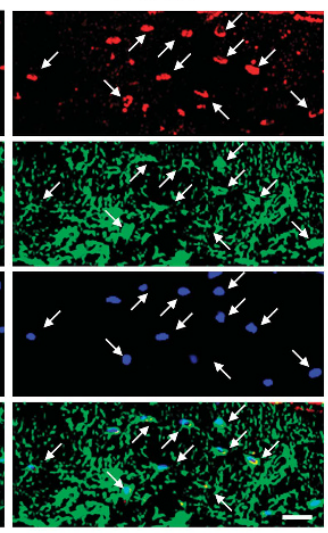

CXXC5 $\%$

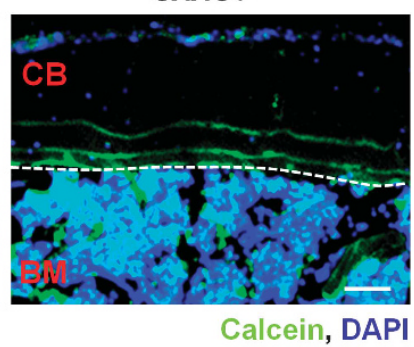

d

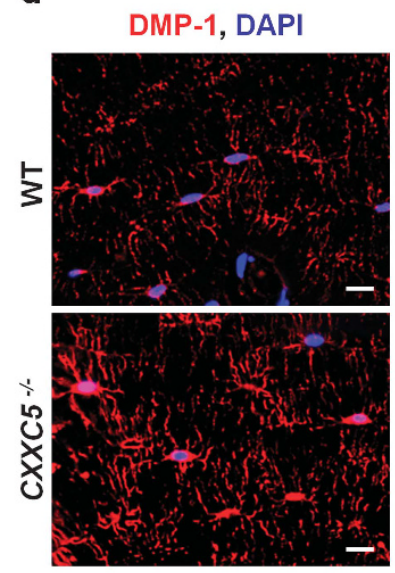

$\mathbf{h}$

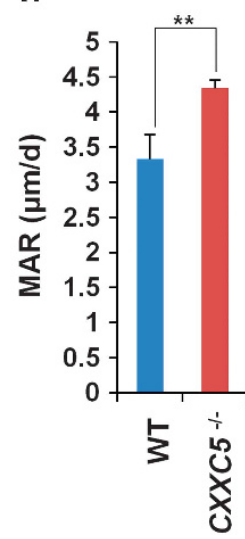

Figure 3 Increase of cortical bone thickness and enhancement of osteocyte network in $C X X C 5^{-1-}$ mice. (a and $\left.\mathbf{b}\right)$ Light microscopic photographs showing H\&E-stained femur longitudinal sections (a; scale bars, $1 \mathrm{~mm}$ ) and magnified images of cortical bones in the boxed area (b; scale bars, $200 \mu \mathrm{m}$ ). (c-f) Horizontal femoral sections of the mice were subjected to immunofluorescence (IF) analyses to visualize $\beta$-catenin (c; red) or DMP-1 (c and d; green and red, respectively). Scale bars, 40 and $20 \mu \mathrm{m}$ for $\mathbf{c}$ and $\mathbf{d}$, respectively. The number and length of osteocyte dendrites are shown in $\mathbf{e}$ and $\mathbf{f}$, respectively. ( $\mathbf{g}$ and $\mathbf{h}$ ) After calcein injection, the prepared femoral sections were subjected to IF analyses to visualize the integrated calcein (green). The dotted lines indicate the cortical bone surfaces. CB, cortical bone. BM, bone marrow (g; scale bar, $100 \mu \mathrm{m})$. The migration distances of integrated calcein from the surface were measured, and the mineral apposition rates (MARs) were calculated ${ }^{24}$ (h). Cell nuclei were counterstained with 4',6-diamidino-2-phenylindole (c, d, g; blue). The data are displayed as the mean \pm S.D. (error bars). Significance was assessed using Kruskal-Wallis test; ${ }^{*} P<0.05$ and ${ }^{* *} P<0.01$

were similar to those of alkaline phosphatase $(A L P)$, an osteoblast marker, ${ }^{14}$ and bone sialoprotein (BSP), a common osteoblast differentiation marker gene, ${ }^{14}$ especially at the late stages of differentiation ( $>14$ days; Figure $4 a$ ). The activation of the $W n t / \beta$-catenin pathway during the differentiation of MC3T3E1 cells into osteoblasts was confirmed by the induction of fibroblast growth factor 18 ( Fgf18), a direct and specific target of the $\mathrm{Wnt} / \beta$-catenin pathway, ${ }^{15}$ and by an increase in the level of $\beta$-catenin (Figures $4 a$ and b). $C X X C 5$ was confirmed as a $\mathrm{Wnt} / \beta$-catenin signaling-inducible gene based on the increase in $C X X C 5$ mRNA in response to $\beta$-catenin overexpression (Supplementary Figure S4A). The induced CXXC5 was localized to the cytosol (Figure 4b). Immunoprecipitation (IP) experiments revealed that endogenous CXXC5 interacted with Dvl in MC3T3E1 cells (Supplementary Figure S4B). The interaction between CXXC5 and Dvl was increased in differentiated MC3T3E1 cells (Supplementary Figure S4B). The in vivo co-localization of these two proteins was observed in periostea of growing young mice, where the co-localization was more remarkable in matured osteoblasts (Supplementary
Figures S4C and D, arrows indicate the direction of osteoblast differentiation). Among the direct transcriptional target genes of $\mathrm{Wnt} / \beta$-catenin signaling, only the expression of the osteogenic gene (Fgf18) but not the oncogenic genes (c-Myc and Cyclin D1) ${ }^{16,17}$ were increased by Wnt3a treatment of pre-osteoblasts (Figure 4c). The Wnt3ainduced increases in ALP and Fgf18 expression levels were suppressed by CXXC5 overexpression (Figure 4c). In addition, Wnt3a-induced $\beta$-catenin accumulation and Wnt/ $\beta$-catenin signaling reporter gene activation were abolished by CXXC5 overexpression in MC3T3E1 cells (Figures 4d and e). Human embryonic kidney 293 (HEK293) cells, a normal cell line with high transfection efficiency, were introduced for further investigations of molecular mechanism. Similar but marginal effects of CXXC5 overexpression were observed in HEK293 cells (Supplementary Figures S5A and B). Previous observations in neuronal stem cells revealed that CXXC5 interacted with $\mathrm{Dvl}$, a positive regulator suppressing the formation of the $\beta$-catenin complex, to inhibit the $\mathrm{Wnt} / \beta$-catenin pathway. ${ }^{9} \mathrm{We}$ found that CXXC5 interacted with Dvl in MC3T3E1 cells as 
a

\section{Days of differentiation (d)}

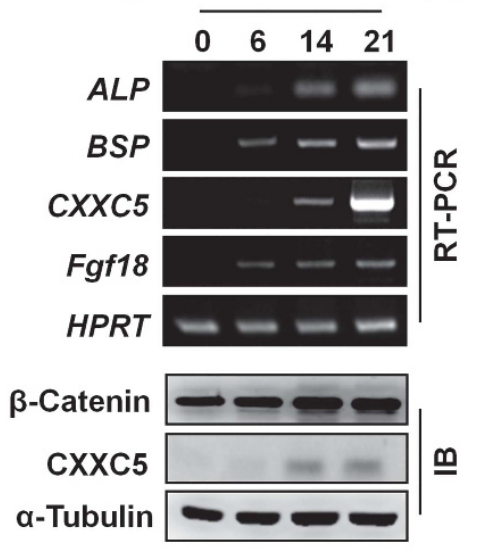

b

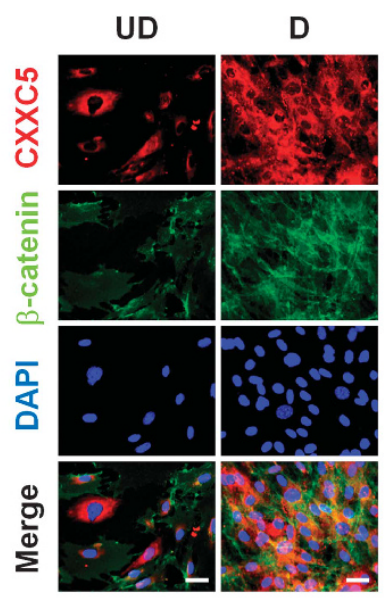

C

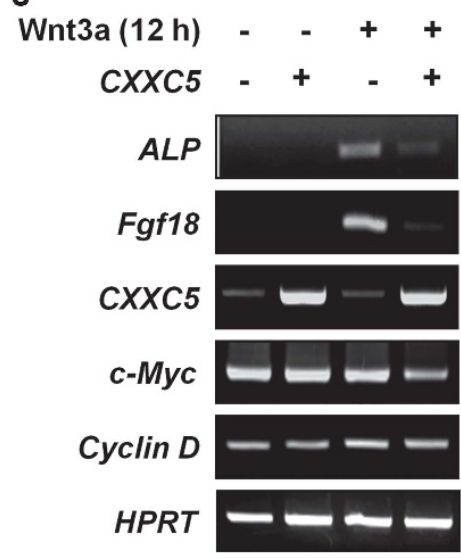

d

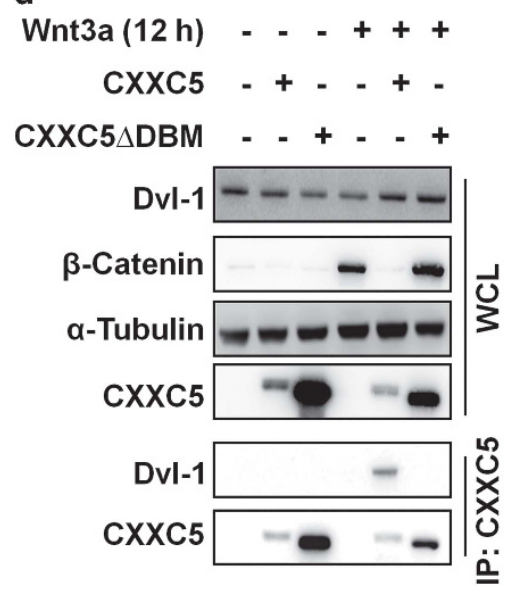

e

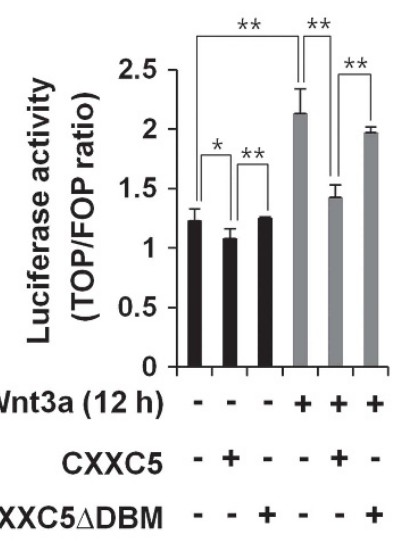

f

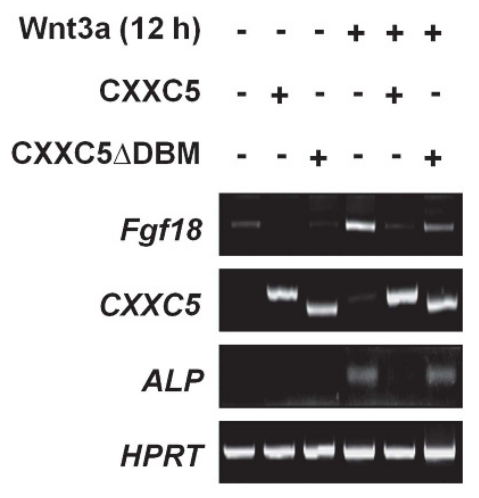

Figure 4 Functional characterization of CXXC5 as a negative-feedback regulator of the Wnt/ $\beta$-catenin pathway and osteoblast differentiation. (a) MC3T3E1 cells were cultured in differentiation media for the indicated number of days. mRNA extracts were analyzed using RT-PCR to detect ALP, BSP, CXXC5, Fgf18 and HPRT. Whole-cell lysates (WCLs) were analyzed using immunoblotting (IB) to detect $\beta$-catenin, CXXC5 and $\alpha$-tubulin. (b) Undifferentiated (UD) and 14-day-differentiated (d) MC3T3E1 cells were subjected to IF analyses to visualize CXXC5 (red) and $\beta$-catenin (green). The cell nuclei were counterstained with 4',6-diamidino-2-phenylindole (blue). Scale bars, $20 \mu \mathrm{m}$. (c) MC3T3E1 cells transfected with pcDNA3.1 control plasmid or pcDNA3.1-CXXC5-Myc were incubated with or without $100 \mathrm{ng} / \mathrm{ml}$ recombinant Wnt3a for $12 \mathrm{~h}$. mRNA extracts were analyzed using RT-PCR to detect ALP, Fgf18, CXXC5, c-Myc, Cyclin D and HPRT. (d-f) MC3T3E1 cells were transfected with pcDNA3.1, pcDNA3.1-CXXC5-Myc or pcDNA3.1-CXXC5 $\triangle$ DBM-Myc. The transfected cells were treated or not treated with $100 \mathrm{ng} / \mathrm{ml}$ of recombinant Wnt3a for $12 \mathrm{~h}$. The WCLs or cell lysates immunoprecipitated with anti-CXXC5 (IP: CXXC5) were analyzed using IB to detect Dvl-1, $\beta$-catenin, $\alpha$-tubulin or CXXC5 (d). For the luciferase reporter assay (e), cells were co-transfected with pTOPFLASH or pFOPFLASH together with pCMV- $\beta$-gal. Activity was calculated as the TOP:FOP ratio, and data shown are the mean \pm S.D. (error bars). The significance was assessed using Student's $t$-test; ${ }^{*} P<0.05$ and ${ }^{* *} P<0.01$. RT-PCR to detect Fgf18, CXXC5, ALP or HPRT is shown in (f)

well, but only when MC3T3E1 cells were treated with Wnt3a (Figure 4d). This Wnt3a-dependent Dvl-CXXC5 interaction was cell-type specific, as it was not observed in HEK293 cells (Supplementary Figure S5C).

CXXC5 possesses a Dvl-binding motif (DBM) at its C terminus, which is an essential motif for Idax to bind with the Dvl PDZ domain ${ }^{18}$ (Supplementary Figure S5D). Overexpression of CXXC5 $\triangle D B M$, which lacks the DBM, did not inhibit Wnt3a-induced activation of the $\mathrm{Wnt} / \beta$-catenin pathway in either MC3T3E1 cells (Figures 4d and e) or HEK293 cells (Supplementary Figure S5E). As expected, CXXC5 DDBM failed to bind Dvl in MC3T3E1 cells, even when the cells were treated with Wnt3a (Figure 4d). Furthermore, CXXC5 DDBM did not affect the Wnt3a-induced increases in the transcriptional activity of the $\mathrm{Wnt} / \beta$-catenin reporter gene (Figure $4 \mathrm{e}$ ) or in ALP and Fgf18 mRNA levels (Figure 4f). In summary,
CXXC5 is a negative-feedback regulator that is induced by $\mathrm{Wnt} / \beta$-catenin signaling during osteoblast differentiation and functions via an osteoblast-specific interaction with Dvl in a Wnt3a-dependent manner.

Blockade of the Dvl-CXXC5 interaction by a competing peptide activates the Wnt/ $\beta$-catenin pathway, and promotes osteoblast differentiation and ex vivo calvaria growth. To investigate whether disruption of the DvI-CXXC5 interaction can activate $\mathrm{Wnt} / \beta$-catenin signaling, a competitor peptide that can bind with Dvl was synthesized (Figure 5a). This peptide consists of a protein transduction domain (PTD; PolyR), ${ }^{19}$ linker, DBM and lysine conjugated to fluorescein isothiocyanate (Figure 5a). In MC3T3E1 cells, nuclear $\beta$-catenin levels and the transcriptional activity of the $\mathrm{Wnt} / \beta$-catenin reporter gene were significantly increased 
a

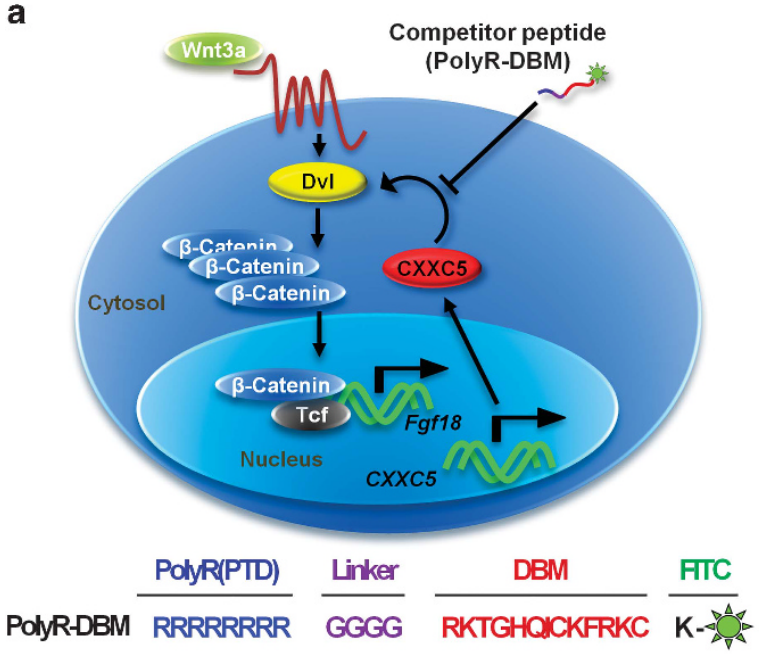

e

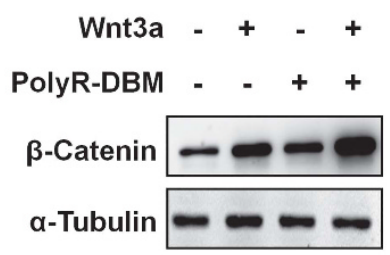

b

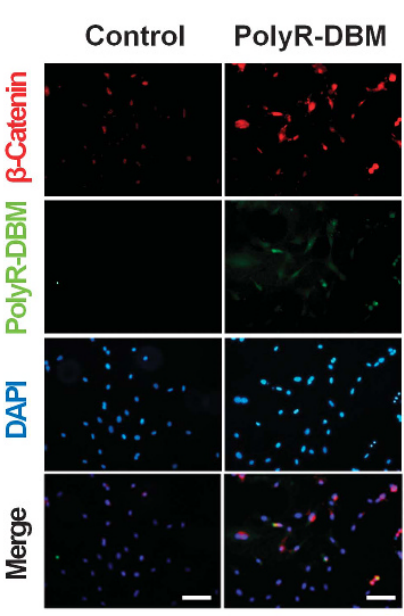

C

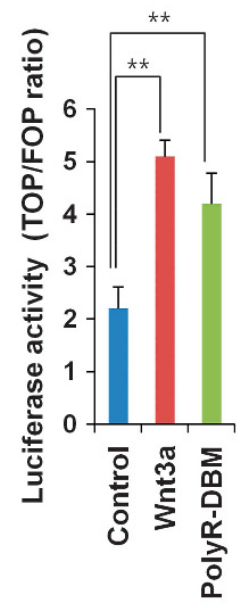

d

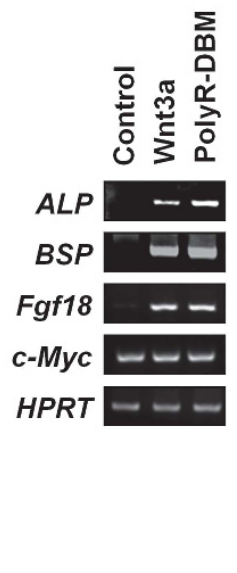

g
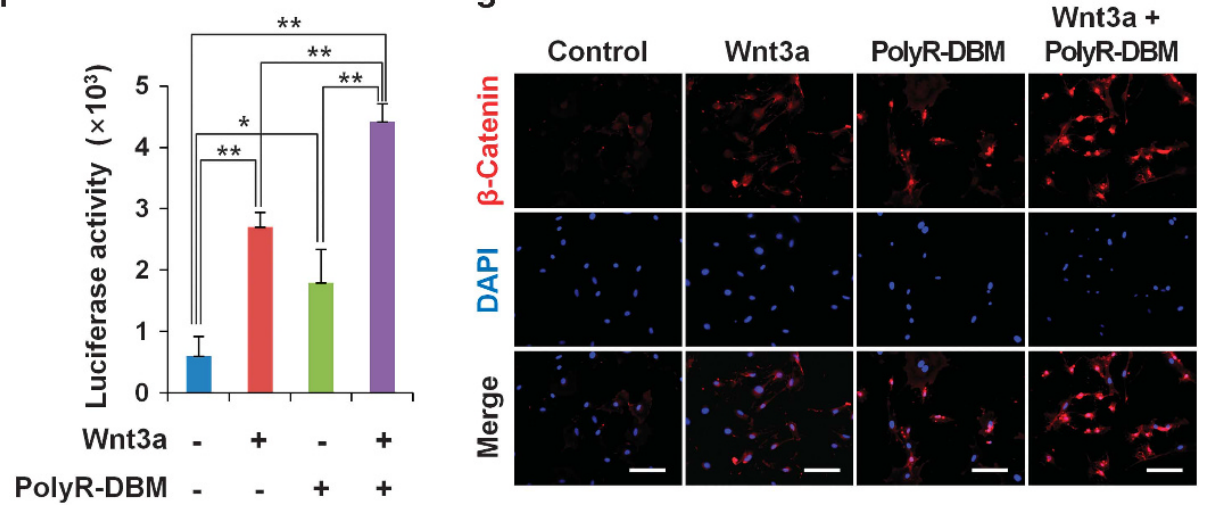

Figure 5 Enhancement of osteoblast differentiation by blockade of Dvl-CXXC5 interaction using competitor peptide and the synergistic effect by co-treatment with recombinant Wnt3a. (a) Disruption of the Dvl-CXXC5 interaction using a competitor peptide, PolyR-DBM induces osteoblast differentiation and bone formation by activation of the Wnt $\beta$-catenin signaling pathway via release of negative-feedback loop. Poly-DBM has a polyglycine linker between PTD and DBM to provide flexibility, and is tagged with fluorescein isothiocyanate on the C-terminal for visualization. (b) $\beta$-catenin (red) was visualized by IF analysis in MC3T3E1 cells following treatment with fluorescein isothiocyanate-tagged PolyR-DBM (green) for $12 \mathrm{~h}$. Nuclei were counterstained with 4',6-diamidino-2-phenylindole (DAPI; blue). Scale bars, $100 \mu \mathrm{m}$. (c) MC3T3E1 cells transfected with pCMV- $\beta$-gal and either pTOPFLASH or pFOPFLASH were treated with $100 \mathrm{ng} / \mathrm{ml}$ recombinant Wnt3a or $5 \mu \mathrm{M}$ PolyR-DBM for 2 days and analyzed using the luciferase reporter assay. (d) MC3T3E1 cells were treated with $100 \mathrm{ng} / \mathrm{ml}$ Wnt3a or $5 \mu \mathrm{M}$ PolyR-DBM for 4 days and were analyzed using RT-PCR to detect ALP, BSP, Fgf18, $c-M y c$ and HPRT. (e and f) The MC3T3E1 cells were incubated in $100 \mathrm{ng} / \mathrm{ml}$ recombinant Wnt3a-containing or non-containing medium with or without $5 \mu \mathrm{M}$ PolyR-DBM for $12 \mathrm{~h}$. The cells were subjected into IB analysis (e), reporter assay (f) and IF analysis for $\beta$-catenin (g). The cell nuclei were counterstained with DAPI (g; blue, scale bars, $100 \mu \mathrm{m})$. The data are the mean \pm S.D. (error bars), and significance was assessed using Student's $t$-test; ${ }^{*} P<0.05$ and ${ }^{* *} P<0.01$.

following loading with PolyR-DBM (Figures $5 \mathrm{~b}$ and $\mathrm{c}$ ). $A L P$, $B S P$ and Fgf18 mRNA levels were also increased after PolyR-DBM treatment (Figure 5d).

The role of CXXC5 as a negative-feedback regulator of the Wnt/ $\beta$-catenin pathway in MC3T3E1 cells was further suggested by the significant synergistic increase in $\beta$-catenin protein levels and $\mathrm{Wnt} / \beta$-catenin signaling reporter activity by treatment of Wnt3a recombinant protein (Wnt3a) with PolyR-DBM compared with treatment of Wnt3a or PolyR-DBM alone (Figures $5 e$ and f). The co-treatment also critically enhanced both the level and the nuclear localization of $\beta$-catenin compared with Wnt3a or PolyRDBM treatment alone (Figure $5 \mathrm{~g}$ ). These results confirm that CXXC5 is a negative-feedback regulator of $\mathrm{Wnt} / \beta$-catenin signaling, and demonstrate that targeting the Dvl-CXXC5 interaction might be a feature of bone anabolic agents.

To show the effects of PolyR-DBM on bone formation, we used the ex vivo calvaria culture system, providing convenient way to access bone formation via the culture of a piece of bone tissue. $^{20}$ Calvariae were cultured with PolyR-DBM and internalization of the peptide into calvariae was confirmed by fluorescence imaging (Figure 6a). $\beta$-Catenin accumulation in calvarial cells was observed in the PolyR-DBM-treated group, which showed activation of $\mathrm{Wnt} / \beta$-catenin signaling in the cells, but not in the untreated group (Figure 6a). PolyR-DBMtreated calvariae exhibited a faster growth rate than calvariae of the non-treated group (Figures $6 b$ and $c$ ). These results show that disruption of Dvl-CXXC5 interaction can promote bone formation of ex vivo-cultured tissues, as well as osteogenic differentiation of osteoblasts.

\section{Discussion}

Two negative regulators of the $\mathrm{Wnt} / \beta$-catenin pathway have been identified as negative-feedback regulators of osteoblast 


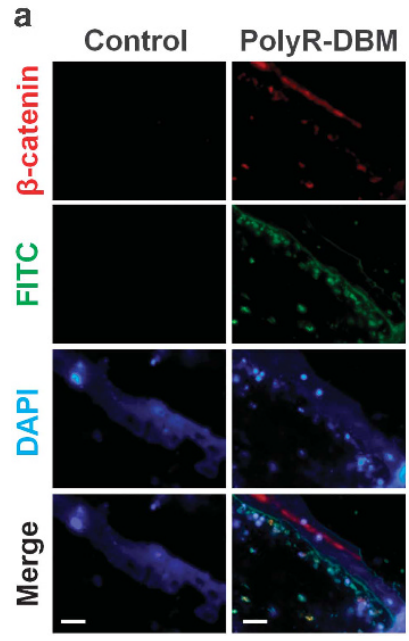

d

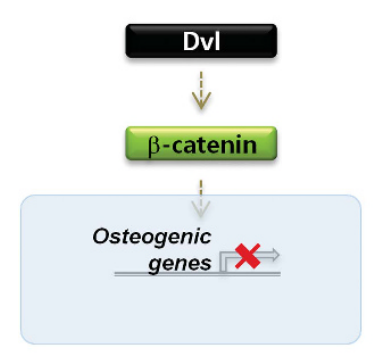

Undifferentiation condition b
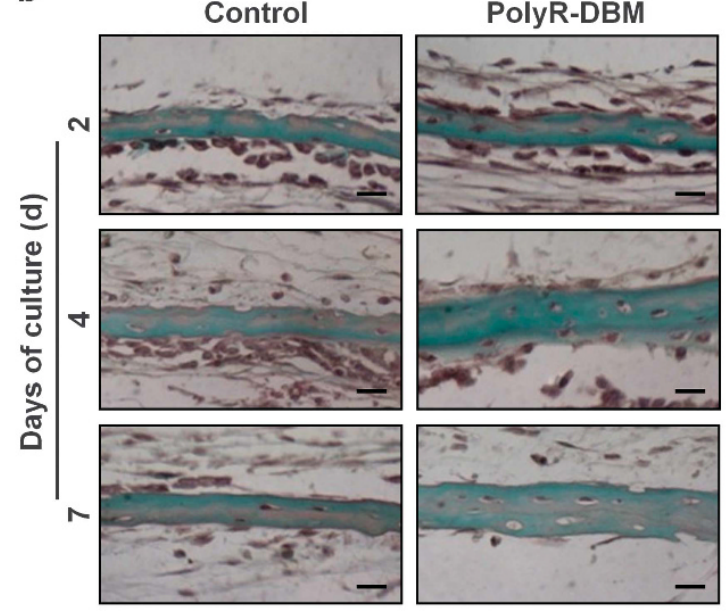

C
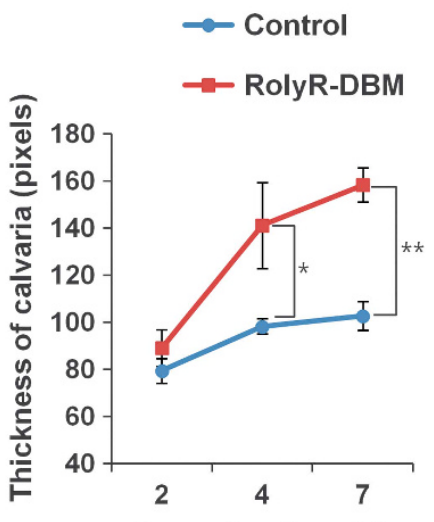

Days of culture (d)

Early Differentiation Stage

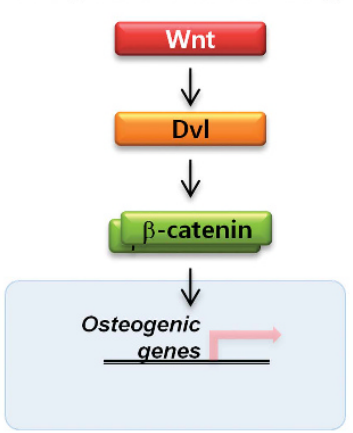

Activation of Wnt/ $\beta$-catenin Signaling
Late Differentiation Stage

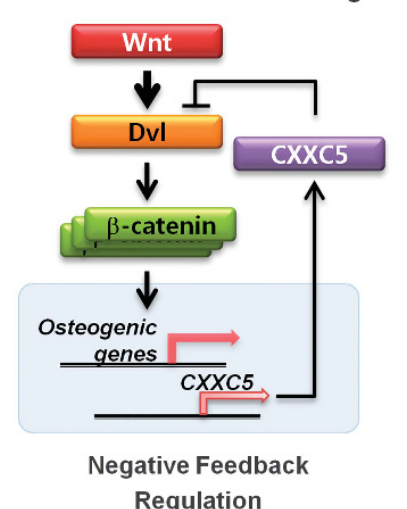

Differentiation Condition

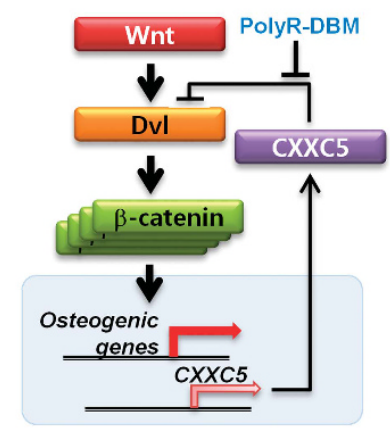

Release of Negative

Feedback Regulation

Figure 6 Enhancement of ex vivo calvaria growth by blockade of DvI-CXXC5 interaction using PolyR-DBM. (a) Calvariae from 4-day-old mice were cultured ex vivo with or without $5 \mu \mathrm{M}$ PolyR-DBM for 2 days. Calvaria sections were stained for IF analysis with anti- $\beta$-catenin (red). Images of calvaria sections show signal from PolyR-DBM (green) and 4',6-diamidino-2-phenylindole (blue). Scale bars, $100 \mu \mathrm{m}$. (b) Representative sections from calvarias from 4-day-old mice cultured for 2, 4 and 7 days with or without $5 \mu \mathrm{M}$ PolyRDBM, and visualized by Safranin $O$ staining. Scale bars, $100 \mu \mathrm{m}$. (c) The thicknesses of calvariae were measured from the images obtained from $\mathbf{b}$ using Image Pro software. The data are the mean \pm S.D. (error bars), and significance was assessed using Student's $t$-test; ${ }^{*} P<0.05$ and ${ }^{* *} P<0.01$. (d) A working model for function of $C X X C 5$ as a negativefeedback regulator of Wnt $/ \beta$-catenin signaling and osteoblast differentiation, and release of the negative-feedback regulation by polyR-DBM. Wnt $\beta$-catenin signaling is activated during pre-osteoblast to osteoblast differentiation, and that induce osteogenic genes. Strong activation of Wnt/ $\beta$-catenin signaling at the late stage of differentiation transcriptionally induces $C X X C 5$ as well as the osteogenic genes, and subsequent binding of the produced CXXC5 to Dvl suppresses Wnt $\beta$-catenin signaling (marked by middle thick arrows). Blockade of the Dvl-CXXC5 interaction with PolyR-DBM releases the negative-feedback regulation, resulting in high activation of Wnt $\beta$-catenin signaling (marked by thick arrows) and subsequent strong induction of osteogenic genes

differentiation and bone formation. Dkk-1, expressed in osteoblasts and osteocytes, prevents Wnt signaling by interacting with Wnt coreceptors. ${ }^{21}$ Sclerostin, expressed in osteocytes exclusively, inhibits osteoblastogenesis and bone formation in vivo by binding to Wnt coreceptors low-density lipoprotein receptor-related protein 5 and $6 .^{22}$ These two extracellular antagonists of the $\mathrm{Wnt} / \beta$-catenin pathway showed bone tissue-specific expression, and bone formation was accelerated following their genetic ablation or functional inhibition. ${ }^{5}$ Here we identified that $\mathrm{CXXC5}$ is an osteoblastspecific interacellular negative-feedback regulator of the Wnt/ $\beta$-catenin pathway and osteoblast differentiation. Although the expression of CXXC5 was observed in several tissues besides bone, the Wnt-dependent interaction of CXXC5 with Dvl was revealed as an osteoblast-specific event.
Our findings show that $\mathrm{CXXC5}$ is transcriptionally induced by the strong activation of $\mathrm{Wnt} / \beta$-catenin signaling at the late stage of osteoblast differentiation and has a role as a negativefeedback regulator through its direct interaction with Dvl (Figure 6d). The release of the negative-feedback regulation by PTD-DBM induced the expression of osteogenic genes promoting osteoblast differentiation. Furthermore, a significant synergic activation of the $\mathrm{Wnt} / \beta$-catenin pathway by co-treatment with Wnt3a and PTD-DBM was revealed by an analysis of pre-osteoblasts. The synergistic effect confirms the role of $\mathrm{CXXC5}$ as a negative-feedback regulator of the $\mathrm{Wnt} / \beta$-catenin pathway in pre-osteoblasts.

In the absence of this negative-feedback regulator, the Wnt/ $\beta$-catenin pathway was critically activated and subsequently increased DMP-1 levels in mouse osteocytes. DMP-1 is 
expressed primarily in osteocytes and promotes the mineralization of matrix and the maturation of osteoblasts into osteocytes. ${ }^{11}$ The increase in DMP-1 levels in femurs of CXXC5 $5^{-1-}$ mice indicates an increase in bone mineral deposition and an acceleration of osteoblast-to-osteocyte transition in the bone. Indeed, $C X X C 5^{-/-}$mice showed high bone mass phenotype, and bone formation rates were increased in these mice as revealed by histomorphometrical analyses. In addition, we observed increases in osteocyte network in the femurs of $C X X C 5^{-1-}$ mice. The network of osteocytes is essential for the function of osteocytes as mechanosensors, which is required for bone maintenance, and an impaired osteocyte network is associated with the failure of bone to repair itself. ${ }^{23}$ These observations indicate that mouse bone quality is improved by $C X X C 5$ knockout.

In conclusion, we characterized CXXC5 as a negativefeedback regulator of $\mathrm{Wnt} / \beta$-catenin signaling in osteoblasts, and propose the Dvl-CXXC5 interaction as an essential mechanism for the negative-feedback regulation of osteoblast differentiation and bone formation.

\section{Materials and Methods}

Cell culture, transfections and reporter assays. MC3T3E1 and HEK293 cells were maintained in $\alpha$-minimal essential medium ( $\alpha$-MEM, Invitrogen, Carlsbad, CA, USA) and Dulbecco's-modified Eagle's medium (Invitrogen), respectively, supplemented with 10\% fetal bovine serum (Invitrogen) and 100 units $/ \mathrm{ml}$ penicillin $\mathrm{G}$ streptomycin (Invitrogen). For osteoblast differentiation, MC3T3E1 cells were grown in $\alpha$-MEM with either ascorbic acid $(50 \mathrm{mg} /$; Sigma, St. Louis, MO, USA) and $\beta$-glycerol-phosphate (10 mM; Sigma) or recombinant Wnt3a $(100 \mathrm{ng} / \mathrm{ml}$; R\&D Systems, Minneapolis, MN, USA). MC3T3E1 cells were transfected with $0.5 \mu \mathrm{g}$ of the vector pcDNA3.1, pcDNA3.1-CXXC5-Myc or pcDNA3.1-CXXC5 $\triangle D B M-M y c$. For the reporter assay, all cells were co-transfected with $0.5 \mu \mathrm{g}$ of pTOPFLASH or pFOPFLASH and $50 \mathrm{ng}$ of $\mathrm{pCMV}-\beta$-gal. The relative luciferase activity is presented as the ratio of pTOPFLASH to PFOPFLASH and normalized to $\beta$-galactosidase activity. The PolyR-DBM peptides described in Figure 5a were synthesized by Peptide $2.0 \mathrm{Inc}$ (Chantilly, VA, USA).

Bone mass measurements, skeletal analyses and bone preparation. The BMD of 11-week-old male mice was determined using a PIXImus II densitometer (GE Healthcare, Piscataway, NJ, USA). The bone phenotypes were measured in male mice to avoid variations resulting from female sex hormones. The mouse femurs were scanned using a CT system (Skyscan 1072, Skyscan, Aartselaar, Belgium). The scanned image data were reconstructed to create 3D images and analyzed using the CT-Analyzing Program (Skyscan). For femoral trabecular bone analysis, the femurs were scanned with a voxel size of $10 \mu \mathrm{m}$ beginning at the end of the growth plate and extending proximally along the diaphysis, and 80 continuous slices (beginning at $0.1 \mathrm{~mm}$ from the most proximal aspect of the growth plate in which both condyles were no longer visible) were selected for analysis. For skeleton staining, the skeletons were fixed in $95 \%$ ethanol and stained with Alizarin red S solution. The images were captured using a SMZ645 Nikon microscope (Nikon, Tokyo, Japan).

Mouse calvaria extraction and ex vivo culture. The parietal bones were isolated from the calvaria of 4-day-old mice as described previously. ${ }^{20}$ For ex vivo culture, each calvaria was placed on a stainless steel mesh bridge support containing $\alpha$-MEM with 10\% FBS (Invitrogen) and 100 units/ml penicillin $\mathrm{G}$ streptomycin (Invitrogen). The medium was exchanged every 2 days. The calvariae were maintained at $37^{\circ} \mathrm{C}$ in a humidified atmosphere of $5 \% \mathrm{CO}_{2}$.

IP and immunoblot analyses. The cells were lysed in RIPA buffer $(50 \mathrm{mM}$ Tris chloride ( $\mathrm{pH} 8.0), 120 \mathrm{mM}$ sodium chloride, $0.5 \%$ (v/v) nonidet P- $40,5 \mu \mathrm{g} / \mathrm{ml}$ leupeptin, $10 \mu \mathrm{g} / \mathrm{ml}$ aprotinin, $50 \mu \mathrm{g} / \mathrm{ml}$ PMSF, $0.2 \mathrm{mM}$ sodium orthovanadate and
$100 \mathrm{mM}$ sodium fluoride) for immunoblot analyses. IP was performed as described previously. ${ }^{10}$ To monitor the interactions between overexpressed proteins, $400 \mu \mathrm{g}$ of lysates of transfected cells were subjected to IP with $2 \mu \mathrm{g}$ of anti-Dvl-1 (3F12; Santa Cruz Biotechnology, Santa Cruz, CA, USA), anti-Myc (9B11; Cell Signaling Technology, Beverly, MA, USA) or anti-CXXC5 antibody. Immunoblot analyses were performed using anti- $\beta$-catenin (E-5; Santa Cruz Biotechnology; $1: 3000$ ), anti-Dvl-1 (1:1000), anti-Myc (Cell Signaling Technology; 1:5000) or anti- $\alpha$-tubulin (Calbiochem, San Diego, CA, USA; 1:5000) followed by incubation with horseradish peroxidase (HRP)-conjugated secondary antibody (Santa Cruz Biotechnology) and visualization using enhanced chemiluminescence (Amersham Biosciences, Piscataway, NJ, USA).

Conventional PCR and reverse transcriptase PCR. The CDNA preparation and reverse transcriptase PCR (RT-PCR) analyses were performed as described previously. ${ }^{14}$ Briefly, RT-PCR was performed with total RNA $(1 \mu \mathrm{g})$, and reverse transcription was performed with the following primers: $B S P$, forward 5'-AATGGAGACGGCGATAGTTC-3' and reverse 5'-GTCCTCATAAGCTCGGTAAG-3'; ALP, forward 5'-GGGACTGGTACTCGGATAACGA-3' and reverse 5'-CTGAT ATGCGATGTCCTTGCA-3'; Fgf18, forward 5'-ACTGCTGTGCTTCCAGGTTC-3' and reverse 5'-CCCAGGACTTGAATGTGCTT-3'; CXXC5, forward 5'-CAGTA GCAGCAGCAGTAACA-3' and reverse 5'-CACTCATCAGTGGCAGGTGT-3'; and hypoxanthine guanine phospho-ribosyltransferase (HPRT), forward $5^{\prime}$-CCTGC TGGATTACATTAAAGCACT-3' and reverse GTCAGGGCATTCCAACAACAAA-3'. G-Taq DNA polymerase (COSMO Genetech, Seoul, Korea) was used for all PCRs, including cloning, genotyping and RT-PCR.

Histochemical analysis. The femurs of 11-week-old mice and the calvariae of 4-day-old mice were fixed in $4 \%$ paraformaldehyde in phosphate-buffered saline. The bones were then decalcified in $10 \mathrm{mM}$ EDTA and embedded in paraffin. Five-micrometer tissue sections were deparaffinized in $100 \%$ xylene followed by re-hydration with sequential $100-50 \%$ ethanol washes. The sections were stained with H\&E or Safranin $\mathrm{O}$ solution. For immunohistochemical analyses, antigen retrieval was performed by incubation in a citrate buffer at $60^{\circ} \mathrm{C}$ for $1 \mathrm{~h}$ or in a buffer containing proteinase $\mathrm{K}$ at $37^{\circ} \mathrm{C}$ for $20 \mathrm{~min}$. To stain with $3,3^{\prime}$-diaminobenzidine (DAB), the sections were treated with $\mathrm{H}_{2} \mathrm{O}_{2}$ to inactivate endogenous tissue peroxidases. The following primary antibodies were used: anti-CXXC5 $(1: 20)$, anti$\beta$-catenin (1:100) and anti-DMP-1 (1:100; Sigma). For immunofluorescence staining and analysis, the sections were incubated with Alexa Fluor 488- or Alexa Fluor 555-conjugated IgG secondary antibody (Molecular Probes, Eugene, OR, USA; 1:200), counterstained with 4',6-diamidino-2-phenylindole (Boehringer Mannheim, Mannheim, Germany) and mounted in Gel/Mount media (Dako, Glostrup, Denmark). An LSM510META confocal microscope (Carl Zeiss, Jena, Germany) was used to capture the fluorescent micrographs of the stained sections. For DAB staining, the sections were incubated with HRP-conjugated IgG secondary antibody (Dako; $1: 200$ ), followed by DAB substrate reagent (Dako). The sections were counterstained with Mayer's hematoxylin (Junsei Chemical, Tokyo, Japan), and light micrographs were captured using a SMZ645 Nikon microscope (Nikon). To minimize variation in the measurement of calvaria thickness, sagittal sections obtained from near a specific position ( $500 \mu \mathrm{m}$ far from midline suture of calvaria) were selected, and the region of interest (ROI) was set on parietal bone nearby the coronal suture. The thicknesses of the bone in the ROI were measured using Image Pro software (Media Cybernetics Inc., Silver Spring, MD, USA).

Statistical analysis. Statistically significant differences were calculated using Student's $t$-test and Kruskal-Wallis test. $P$-values $<0.05$ were considered significant $\left({ }^{*} P<0.05,{ }^{* *} P<0.01\right.$ and $\left.{ }^{* * *} P<0.001\right)$. For all statistical analyses, Statistical Package for the Social Sciences (SPSS 21, IBM, Chicago, IL, USA) statistical program was used.

\section{Conflict of Interest}

The authors declare no conflict of interest.

Acknowledgements. We thank Sean Bong Lee and Ho Jin Lee for their helpful discussion and comments on the manuscript. This work was funded by the Ministry of Future Creation and Science (MFCS) of Korea; Translational Research Center for Protein Function Control (2009-0083522), Mid-career Researcher Program 
(2012-010285) and Stem Cell Research Project (2010-0020235). This work was also supported by the Ministry of Knowledge Economy (through the Korea Research Institute of Chemical Technology (SI-0905, SI-1005, SI-1105, SI-1205 and SI-1304)). $\mathrm{H}-\mathrm{YK}, \mathrm{J}-\mathrm{YY}, \mathrm{S}-\mathrm{HL}$ and K-WC were supported by a BK21 studentship from the NRF and the MFCS.

1. Eriksen EF. Cellular mechanisms of bone remodeling. Rev Endocr Metab Disord 2010; 11: 219-227.

2. Long F. Building strong bones: molecular regulation of the osteoblast lineage. Nat Rev Mol Cell Biol 2012; 13: 27-38.

3. Rachner TD, Khosla S, Hofbauer LC. Osteoporosis: now and the future. Lancet 2011; 377 : 1276-1287.

4. Regard JB, Zhong Z, Williams BO, Yang Y. Wnt signaling in bone development and disease: making stronger bone with Wnts. Cold Spring Harb Perspect Biol 2012; 4: 1-17.

5. Hoeppner LH, Secreto FJ, Westendorf JJ. Wnt signaling as a therapeutic target for bone diseases. Expert Opin Ther Targets 2009; 13: 485-496.

6. Ravasi T, Huber T, Zavolan M, Forrest A, Gaasterland T, Grimmond S et al. Systematic characterization of the zinc-finger-containing proteins in the mouse transcriptome. Genome Res 2003; 13: 1430-1442.

7. Pendino F, Nguyen E, Jonassen I, Dysvik B, Azouz A, Lanotte M et al. Functional involvement of RINF, retinoid-inducible nuclear factor (CXXC5), in normal and tumoral human myelopoiesis. Blood 2009; 113: 3172-3181.

8. Kim HY, Yang DH, Shin SW, Kim MY, Yoon JH, Kim S et al. CXXC5 is a transcriptional activator of Flk-1 and mediates bone morphogenic protein-induced endothelial cell differentiation and vessel formation. FASEB J 2014; 28: 615-626.

9. Andersson T, Södersten E, Duckworth JK, Cascante A, Fritz N, Sacchetti P et al. CXXC5 is a novel BMP4-regulated modulator of Wnt signaling in neural stem cells. J Biol Chem 2009; 284: $3672-3681$.

10. Hino S, Kishida S, Michiue T, Fukui A, Sakamoto I, Takada S et al. Inhibition of the Wnt signaling pathway by Idax, a novel Dvl-binding protein. Mol Cell Biol 2001; 21: 330-342.
11. Qin C, D'Souza R, Feng JQ. Dentin matrix protein 1 (DMP1): new and important roles for biomineralization and phosphate homeostasis. J Dent Res 2007; 86: 1134-1141.

12. Bonewald LF, Johnson ML. Osteocytes, mechanosensing and Wnt signaling. Bone 2008; 42 : $606-615$.

13. Stuart AJ, Smith DA. Use of the fluorochromes xylenol orange, calcein green, and tetracycline to document bone deposition and remodeling in healing fractures in chickens. Avian Dis 1992; 36: 447-449.

14. Wang D, Christensen K, Chawla K, Xiao G, Krebsbach PH, Franceschi RT et al. Isolation and characterization of MC3T3-E1 preosteoblast subclones with distinct in vitro and in vivo differentiation/mineralization potential. J Bone Miner Res 1999; 14: 893-903.

15. Reinhold MI, Naski MC. Direct interactions of Runx2 and canonical Wht signaling induce FGF18. J Biol Chem 2007; 282: 3653-3663.

16. He TC, Sparks AB, Rago C, Hermeking H, Zawel L, da Costa LT et al. Identification of C-MYC as a target of the APC pathway. Science 1998; 281: 1509-1512.

17. Shtutman M, Zhurinsky J, Simcha I, Albanese C, D'Amico M, Pestell R et al. The cyclin D1 gene is a target of the beta-catenin/LEF-1 pathway. Proc Natl Acad Sci USA 1999; 96: 5522-5527.

18. London TB, Lee HJ, Shao Y, Zheng J. Interaction between the internal motif KTXXXI of Idax and mDvl PDZ domain. Biochem Biophys Res Commun 2004; 322: 326-332.

19. Matsushita M, Matsui H. Protein transduction technology. J Mol Med 2005; 83: 324-328.

20. Reynolds JJ, Minkin C, Morgan DB, Spycher D, Fleisch H. The effect of two diphosphonates on the resorption of mouse calvaria in vitro. Calcif Tissue Res 1972; 10: 302-313.

21. Canalis E, Giustina A, Bilezikian JP. Mechanisms of anabolic therapies for osteoporosis. N Engl J Med 2007; 357: 905-916.

22. Li X, Zhang Y, Kang H, Liu W, Liu P, Zhang J et al. Sclerostin binds to LRP5/6 and antagonizes canonical Wnt signaling. J Biol Chem 2005; 280: 19883-19887.

23. Vashishth D, Verborgt O, Divine G, Schaffler MB, Fyhrie DP. Decline in osteocyte lacunar density in human cortical bone is associated with accumulation of microcracks with age. Bone 2000; 26: 375-380.

24. Parfitt AM, Drezner MK, Glorieux FH, Kanis JA, Malluche H, Meunier PJ et al. Bone histomorphometry: standardization of nomenclature, symbols, and units. Report of the ASBMR Histomorphometry Nomenclature Committee. J Bone Miner Res 1987; 2: 595-610.

Supplementary Information accompanies this paper on Cell Death and Differentiation website (http://www.nature.com/cdd) 